Canadian Journal of Civil Engineering

Canadian

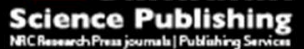

Revue canadienne de génie civil

\title{
INSTABILITY OF UNBOUNDED TRANSVERSE MIXING LAYER IN SHALLOW WATERS
}

\begin{tabular}{|r|l|}
\hline Journal: & Canadian Journal of Civil Engineering \\
\hline Manuscript ID & cjce-2016-0018.R1 \\
\hline Manuscript Type: & Article \\
\hline Date Submitted by the Author: & 11 -Mar-2016 \\
\hline Keyword: List of Authors: & $\begin{array}{l}\text { Karimpour, Shooka; McGill University, Civil Engineering and Applied } \\
\text { Mechanics } \\
\text { Chu, Vincent H.; Dept. of Civil Engineering and Applied Mechanic, }\end{array}$ \\
\hline & $\begin{array}{l}\text { Shear Instabilities, Shallow Flow, Waves in Currents, Direct Numerical } \\
\text { Simulations }\end{array}$ \\
\hline & \\
\hline $\begin{array}{l}\text { Note: The following files were submitted by the author for peer review, but cannot be converted to } \\
\text { PDF. You must view these files (e.g. movies) online. }\end{array}$ \\
\hline Figs.zip
\end{tabular}




\section{INSTABILITY OF UNBOUNDED}

TRANSVERSE MIXING LAYER IN

\section{SHALLOW WATERS}

Shooka Karimpour and Vincent H. Chu

Shooka Karimpour ${ }^{1}$ and Vincent H. Chu. Department of Civil Engineering and Applied Mechanics, McGill University, Montreal, QC, Canada, H3A 0C3

${ }^{1}$ Corresponding author (e-mail: Shooka.Karimpour@mcgill.ca, Phone: +1-514-398-1609). 
Abstract: This paper examines the role of waves in the development of the instability of an unbounded transverse mixing layer in shallow waters. In supercritical state waves become significant. The matching of the wave-like asymptotic solution in the far field to the solutions near the inflection and the returning surfaces poses a difficult mathematical problem. We therefore conducted numerical simulations to find solutions directly from the shallow-water equations. The simulations found that the growing waves in the unstable current had a consistent structure throughout the linear stage of the instability's development. The excitation, reflection and transmission of waves produced a modulating instability that maintained a consistent structure as the amplitude of the instability increased over many orders of magnitude. This role of the waves on the supercritical instability, as delineated by our numerical simulations, is not solely describable by the classical analytical method.

Key words: Shear Instabilities, Shallow Flow, Waves in Currents, Direct Numerical Simulations. 


\section{Introduction}

The transverse mixing layer in shallow waters is a shear flow developed between free streams of different velocities. The mixing layer in deep water is unstable according to Rayleigh's Inflexion Point Theorem (Rayleigh 1880). In shallow waters, the instability produces gravity waves in the mixing layer, while the instability is in turn affected by the reflection and transmission of the waves. In his formulation, Balmforth (1999) defined the instability in shallow waters at the inflection point of the base flow, and at the returning surfaces where the flow velocity matches the speed of the gravity waves. Takehiro \& Hayashi (1992) delineated the role of waves on the instability of linear shear flow in shallow waters by the over-reflection and transmission of waves through the returning surface. Waves are known to similarly affect the instability of other shear flows that admit waves. These include the problems that occur in compressible flow (Blumen et al. 1975; Sandham \& Reynolds 1990; Mack 1990), in rotating shear flow (Lindzen et al. 1980; Maslowe 1991) and in gravity stratified flow (Lindzen \& Barker 1985). For the unbounded shear flow, Maslowe (1991) matched the wave-like asymptotic solution in the far field to the solutions near the inflection and the returning surfaces via a radiation boundary condition to allow the waves to escape. Sandham \& Reynolds (1990) found the growth rate of the instability in the mixing layer up to a Mach number $\mathrm{Ma}_{\mathrm{c}}=1.2$, using the normal-mode method. The simulation for the unbounded mixing layer described in this paper was conducted without making a priori assumptions for the normal mode. The direct numerical simulation is intended to provide some of the wave-and-current interaction details that are beyond those that are obvious from the normal-mode solution.

\section{Unbounded Mixing Layer in Shallow Waters}

As shown in Figure 1, in our simulation the base flow of the unbounded mixing layer is the hyperbolic-tangent (TANH) velocity profile:

$U=\frac{1}{2}\left(U_{1}+U_{2}\right)+\frac{1}{2}\left(U_{1}-U_{2}\right) \tanh \frac{2 y}{\delta_{\omega}}$

The velocities in the free stream are $U_{1}$ and $U_{2}$. Simulation for the instabilities starts with a small perturbation $\left(h^{\prime}, u^{\prime}, v^{\prime}\right)$ to the base flow $(H, U, 0)$. The evolution of the disturbance $\left(h^{\prime}, u^{\prime}, v^{\prime}\right)$ with time is determined from the numerical solution of the following shallow-water equation for $h=H+h^{\prime}$, 
$u=U+u^{\prime}, v=v^{\prime}:$

$\frac{\partial h}{\partial t}+\frac{\partial u h}{\partial x}+\frac{\partial h v}{\partial y}=0$

$\frac{\partial u}{\partial t}+u \frac{\partial u}{\partial x}+v \frac{\partial u}{\partial y}=-g \frac{\partial h}{\partial x}$

$\frac{\partial v}{\partial t}+u \frac{\partial v}{\partial x}+v \frac{\partial v}{\partial y}=-g \frac{\partial h}{\partial y}$

where $g$ is the gravity. The simulations are conducted using a fifth-order WENO scheme for the spatial interpolation on a staggered grid and a fourth-order Runge-Kutta method for the time integration. The computational domain is defined by the periodic boundary condition over one wave length in the longitudinal direction from $x=0$ to $x=\lambda_{x}$, and the radiation boundary condition at $y=y^{-}$ and $y=y^{+}$. Simulations for the shear instability are conducted using a computational domain of $y^{+}=-y^{-}=3 \lambda_{x}$ and 256 nodes over the wave length $\lambda_{x}$. The calculations are shown to converge, reaching an accuracy of 4 significant digits for the exponential growth rate of the disturbance. The numerical procedure and the convergence of the simulation to true solution are given in recent papers by Karimpour \& Chu (2015a) and Karimpour \& Chu (2015b). In the present simulations, the initial disturbance is a small depth fluctuation $h^{\prime} / \delta_{\omega}=10^{-10} \sin \left(2 \pi / \lambda_{x}\right)$ specified along a narrow strip in the central region between $y=-\lambda_{x} / 64$ and $y=+\lambda_{x} / 64$ ( $h^{\prime}=0$ elsewhere). The velocity scale is $\left(U_{1}-U_{2}\right)$. The length scale is the vorticity thickness $\delta_{\omega}=\left(U_{1}-U_{2}\right) / \hat{U}_{y}$, where $\hat{U}_{y}$ is the shearing rate at the inflection point. The development of the instability depends on the wave number of the disturbance $k_{x}=2 \pi / \lambda_{x}$, and the convective Froude number $\mathrm{Fr}_{\mathrm{c}}$

$\operatorname{Fr}_{\mathrm{c}}=\frac{U_{1}-U_{2}}{2 \sqrt{g H}}$

where $\sqrt{g H}$ is the speed of the gravity waves. The classical analysis is based on the assumption of the normal mode

$\left[h^{\prime}, u^{\prime}, v^{\prime}\right]=[\hat{h}(y), \hat{u}(y), \hat{v}(y)] \exp i\left(k_{x} x-\Omega t\right)$. 
Implicit in the normal-mode approach is the requirement of a time-independent relation of the real and imaginary parts of $\Omega$ with $k_{x}$ and $\mathrm{Fr}_{\mathrm{c}}$ (Criminale et al. 2003; Schmid \& Henningson 2012). The real part of $\Omega$ is the frequency of the waves $\omega=\Re(\Omega)$. The imaginary part $\Im(\Omega)$ is the fractional growth rate

$\alpha=\frac{1}{h^{\prime}} \frac{d h^{\prime}}{d t}=\frac{1}{u^{\prime}} \frac{d u^{\prime}}{d t}=\frac{1}{v^{\prime}} \frac{d v^{\prime}}{d t}=\frac{d\left[\ln h^{\prime}\right]}{d t}=\frac{d\left[\ln u^{\prime}\right]}{d t}=\frac{d\left[\ln v^{\prime}\right]}{d t}$

In the direct numerical simulations, the fractional growth rate $\alpha$ is calculated from the growth of the disturbance's kinetic energy $K^{\prime}=\frac{1}{2}\left(u^{\prime 2}+v^{\prime 2}\right)$ as follows:

$\alpha=\frac{d\left[\ln \sqrt{\overline{K^{\prime}}}\right]}{d t}$ where $\overline{K^{\prime}}=\frac{1}{\lambda_{x}} \int_{0}^{\lambda_{x}} K^{\prime} d x, \quad \overline{\overline{K^{\prime}}}=\frac{1}{\delta_{\omega}} \int_{y^{-}}^{y^{+}} \overline{K^{\prime}} d y$

The single over-line is the operator for the averaging from $x=0$ to $x=\lambda_{x}$. The double over-line is the operator for further averaging from $y=y^{-}$to $y=y^{+}$. The characteristic velocity $\sqrt{\overline{\overline{K^{\prime}}}} /\left(U_{1}-U_{2}\right)$ grows exponentially with time in an unstable shear flow. The slope of the lines on a semi-logarithmic scale with time $t \hat{U}_{y}$ in Figure 2 is the fractional growth rate $\alpha$. For the subcritical instability shown in Figure 2 (a) for $\operatorname{Fr}_{\mathrm{c}}=0.95$ and $k_{x}=0.4$, the fractional growth is the constant rate $\alpha / \hat{U}_{y}=$ 0.0407 defined by the slope of the straight line in the figure. For the supercritical instabilities shown in Figure 2 (b) for $\mathrm{Fr}_{\mathrm{c}}=1.2, k_{x}=0.35$ and (c) for $\mathrm{Fr}_{\mathrm{c}}=1.4, k_{x}=0.35$, the fractional growth rate is not constant, but modulates about the average slopes $\alpha / \hat{U}_{y}=0.01365$ and 0.0094 respectively. Table 1 gives the amplitude of the disturbance from the $t_{b} \hat{U}_{y} \simeq 20,90$ and 250 to the $t_{e} \hat{U}_{y} \simeq 500,1450$ and 2250 , for the cases (a), (b) and (c) respectively, when the fractional growth is maintaining a consistent rate. Over these linear stages of the instability's development, the amplitudes of the disturbance are magnified millions of times, but remain sufficiently small for the nonlinear terms to be negligible. The instability of shear flow in gas is a closely analogous problem affected by the waves. Sandham \& Reynolds (1990) determined the fractional rates $\alpha$ for ideal gas with the TANH velocity profile using the classical method. Figure 3 (a) compares the rates obtained from the present simulation for two convective Froude numbers, $\mathrm{Fr}_{\mathrm{c}}=0.1$ and 0.8 , with the analogous results obtained by Sandham $\&$ Reynolds (1990) for the convective Mach number $\mathrm{Ma}_{\mathrm{c}}=0.01$ and 0.8 over the range of wave number $k_{x}=0$ to 2.0. The shallow-water equations are identical to the gas-dynamic equations when value of 
the specific heat ratio of the gas is 2 (Liggett 1994). In the analogous problem in gas, the speed of the sound $a$ defines the convective Mach number $\mathrm{Ma}_{\mathrm{c}}=\left(U_{1}-U_{2}\right) /(2 a)$. The supercritical instability is not an eigenvalue problem because the fractional growth rate is not a constant value that can be determined as an eigenvalue. Nevertheless, the rate obtained by Sandham \& Reynolds (1990) for Mach number $\mathrm{Ma}_{\mathrm{c}}=1.2$, shown as a dashed line in Figure $3(\mathrm{~b})$, compares well with our direct numerical simulation results for $\mathrm{Fr}_{\mathrm{c}}=1.2$. The growth of the supercritical instability may be small, but is important in the final developement of the sheart flows. For the supercritical instability of $\operatorname{Fr}_{\mathrm{c}}=1.4$ and $k_{x}=0.35$, the growth is maintained at the averaged rate of $\alpha / \hat{U}_{y}=0.0094$ as the disturbance's amplitude is amplified $1.5 \times 10^{8}$ times, from $\sqrt{\overline{\overline{K^{\prime}}}} b /\left(U_{1}-U_{2}\right)=2.0 \times 10^{-9}$ to $\sqrt{\overline{\overline{K^{\prime}}}}$ e $/\left(U_{1}-U_{2}\right)=0.313$ (see Table 1). This consistently slow growth, with modulation leading to a huge amplification, occurs entirely in the linear stage of the instability's development. The direct numerical simulation is one approach among others that have been developed to study linear instability problems without the eigenvalue (see Trefethen et al. 1993; Criminale et al. 2003; Schmid \& Henningson 2012).

\section{Constant versus Consistent Structure of the Instability}

Figure 4 shows the contrast between the constant structure of the subcritical instability of $\operatorname{Fr}_{\mathrm{c}}=0.1$ in (a) and the consistent structure of the supercritical instability of $\mathrm{Fr}_{\mathrm{c}}=1.4$ in (b) and (c). The vorticity fluctuation $\zeta^{\prime}=\partial_{x} v^{\prime}-\partial_{y} u^{\prime}$ is plotted on the left-hand side of the figure for the profiles at four longitudinal positions: $x=\frac{1}{4} \lambda_{x} ; x=\frac{1}{2} \lambda_{x} ; x=\frac{3}{4} \lambda_{x}$; and $x=\lambda_{x}$. On the right-hand side of the figure are the profiles of the Reynolds stresses $\overline{u^{\prime} u^{\prime}}$ and $\overline{v^{\prime} v^{\prime}}$ averaged over the wave length $\lambda_{x}$ between the periodic boundary conditions.

The most unstable flow of the subcritical instability with $\mathrm{Fr}_{\mathrm{c}}=0.1$ occurs at the wave number $k_{x}=0.9$. The vorticity fluctuation $\zeta^{\prime} \delta_{\omega} / \sqrt{\overline{\overline{K^{\prime}}}}$ profiles and the Reynolds stresses $\overline{u^{\prime} u^{\prime}} / \overline{\overline{K^{\prime}}}$ and $\overline{u^{\prime} u^{\prime}} / \overline{\overline{K^{\prime}}}$ profiles for this $\mathrm{Fr}_{\mathrm{c}}=0.1$ are shown in the top row of the figure. With the proper normalization, these profiles of the growing waves are unchanged; the constant structure is maintained throughout the development as the disturbance is magnified millions and million of times from the beginning of time $t_{b} \hat{U}_{y}$ to the end of time $t_{e} \hat{U}_{y}$ (see Table 1 ). Such structure is constant for exactly the same reason that the normal mode is the admissible solution for the eigenvalue problem.

The unbounded mixing layer becomes supercritical when the current speed relative to the free streams exceeds the speed of the gravity waves; that is, when $\left(U_{1}-U\right)>\sqrt{g H}$ and $\left(U-U_{2}\right)>\sqrt{g H}$. 
The instability in the supercritical state is not constant but has a consistent structure modulating over the period $T_{M}$. Figure $4\left(b_{1}\right)$ and $\left(b_{2}\right)$ show the profiles of the consistent structure of the instability for $\mathrm{Fr}_{\mathrm{c}}=1.4$ and $k_{x}=0.35$. The most unstable flow for this convective Froude number occurs at the wave number $k_{x}=0.35$. The profiles are shown at time intervals of $\frac{1}{2} T_{M}$ over the modulation period. As given in Table 1, the modulation period of this supercritical instability is $T_{M} \hat{U}_{y}=55$, which is approximately equal to twice the period of the gravity wave $T \hat{U}_{y}=27$.

\subsection{Anisotropic Structure that Depends on Convective Froude Number}

The presence of the waves increases with the convective Froude number $\mathrm{Fr}_{\mathrm{c}}$. Figure 7(a) shows the disturbance's potential energy $P^{\prime}=\frac{1}{2} g^{\prime} h^{\prime 2}$ relative to the disturbance's kinetic energy. The value $\overline{\overline{P^{\prime}}} / H \overline{\overline{K^{\prime}}}$ is negligible for the subcritical flow up to $\operatorname{Fr}_{\mathrm{c}}=0.2$. It increases almost linearly with $\mathrm{Fr}_{\mathrm{c}}$ and then reaches a value of about $\overline{\overline{P^{\prime}}} / H \overline{\overline{K^{\prime}}} \simeq 0.75 \pm 0.25$ for the supercritical flow. The potential energy of the disturbance is comparable to the disturbance's kinetic energy during the development of the supercritical instability. As waves become progressively more prominent, the structure of the instability modified by the presence of the waves increasingly becomes anisotropic. The lateral component of the velocity fluctuations $v^{\prime} v^{\prime}$ relative to the longitudinal component $u^{\prime} u^{\prime}$ decreases with the convective Froude number as shown in Figure 7(b). The momentum exchange by the Reynolds stress $u^{\prime} v^{\prime}$ also decreases with the convective Froude number as shown in Figure 7(c).

\subsection{Trapped Waves between the Returning Surfaces}

The most remarkable feature of the supercritical instability occurs at the returning surfaces where waves change direction. The returning surfaces are identified by the sharp changes in momentum and vorticity gradients shown in Figure $1(b$ and $c)$ and Figure $4\left(b_{1}\right.$ and $\left.b_{2}\right)$. Previous studies (Balmforth 1999; Takehiro \& Hayashi 1992) showed that the returning surfaces occur at locations where the current speed relative to the free streams matches the speed of the gravity waves. There are two returning surfaces in the unbounded mixing layer. On side 1 of the mixing layer at $y=y_{r 1}$, the velocity relative to the free stream at the returning surface is

$U_{1}-U_{r 1}=\sqrt{g H}$ 
On side 2 at $y=y_{r 2}$, the relative velocity is

$U_{r 2}-U_{2}=\sqrt{g H}$

These locations are marked by the pair of dotted dashed lines in Figure 1 and Figure 4 which can be seen to coincide closely with the sharp changes in the vorticity gradient. Intense wave activity occurs in the central region between the returning surfaces. The lateral $\overline{v^{\prime} v^{\prime}}$ component of the Reynolds stresses in the region however is negligibly small compared with its $\overline{u^{\prime} u^{\prime}}$ component. The waves are trapped between the returning surface travelling primarily in the longitudinal direction. The directions of the waves however change rapidly across the returning surfaces.

\subsection{Angle of the Oblique Waves in the Free Streams}

Outside the trapped region, the waves are oriented at an oblique angle $\theta$ from the longitudinal $x$ direction. As shown in Figure 1, the wave number vector $k$ on the $x-y$ plane has two components; the component $k_{x}$ is specified while the component $k_{y}$ is determined from the numerical simulation. Figure 5 (a), (b), (c) and (d) show the lateral profiles of the oblique waves in the free stream at onequarter intervals over the modulation period. The $y$-component of the wave length in the free stream $\lambda_{y a}$ is determined from the numerical simulation over the expanded region of these profiles. Despite the modulation, the same $\lambda_{y a}$ is determined from the profiles (a), (b), (c) and (d) over the modulation period. The oblique angle $\theta$ of the waves in the free stream is therefore uniquely determined from the numerical simulation using the formula

$\sin \theta=\frac{k_{x}}{k}=\frac{k_{x}}{\sqrt{k_{x}^{2}+k_{y a}^{2}}}$.

The $y$-component of the wave-number vector in the free stream is $k_{y a}=2 \pi / \lambda_{y a}$.

\subsection{Location of the Wave Maker}

The oblique waves in the free streams are the product of a moving wave maker. The speed of the wave maker is critically dependent on the location of the wave maker. One hypothesis is to assume the wave maker is located at the other side of the returning surface, as described in Figure 1. The oblique waves in side 1 of the free stream are produced by the over-reflection at the returning surface on side 
2. On the other hand, the oblique waves in side 2 of the free stream are produced by the over-reflection at the returning surface on side 1 . According to this hypothesis, the oblique angle $\theta$ of the waves in the free stream would depend on $\mathrm{Fr}_{\mathrm{c}}$ as follows:

$\sin \theta=\frac{\sqrt{g H}}{U_{1}-U_{2}+\sqrt{g H}}=\frac{1}{2 \mathrm{Fr}_{\mathrm{c}}-1}$.

Figure 6(a) shows the agreement of the oblique angle obtained from the simulations with the formula derived from the hypothesis for the two Froude numbers over a range of different wave numbers. The abscissa in the figure is the $\sin \theta$ determined by Equation 11 from the direct numerical simulation, while the ordinate in the same figure is the $\sin \theta$ determined by Equation 12 derived from the above hypothesis. The other possible location of the wave maker is the point of inflection. Although the inflection point is only a short distance from the returning surfaces, the oblique angle of the wave in the free stream would be determined by a very different formula. If the wave maker were assumed to be located at the inflection point, where the speed is $\frac{1}{2}\left(U_{1}+U_{2}\right)$, the oblique angle would be

$\sin \theta=\frac{\sqrt{g H}}{\frac{1}{2}\left(U_{1}-U_{2}\right)}=\frac{1}{\operatorname{Fr}_{\mathrm{c}}}$.

Figure 6 (b) shows how the $\sin \theta$ in the ordinate is calculated by the incorrect formula. Clearly, the waves are not generated at the inflection point; that is why Equation 13 does not agree with the simulation.

\subsection{Transmitted Waves in the Free Streams}

The waves beyond the returning surfaces in the free streams are not small. Waves that are generated on one returning surface are partially transmitted through the other returning surface. The transmitted waves reduce in amplitude with distance away from the returning surface, as shown in Figure 5, because the strength of the wave maker is growing exponentially at a modulating rate. The envelope of the modulation is the thin dot-dot line in the figure. It defines the amplitudes of the crest of the waves $A(t, y)$, which is a function of the time $t$ and the lateral position $y$. The waves of this amplitude are produced at the wave maker at an earlier time $t_{\text {wavemaker }}=t-t_{\text {travel }}$. The travel time from the wave maker located at $y_{\text {wavemaker }}$ to the waves at position $y$ is $t_{\text {travel }}=\left(y-y_{\text {wavemaker }}\right) \cos \theta / \sqrt{g H}$. The ratio of the amplitude of these waves $A(t, y)$, and the amplitude of the waves at the wave maker 
$A_{\text {wavemaker }}\left(t_{\text {wavemaker }}, y\right)$ that produce these waves at the earlier time $t_{\text {wavemaker }}$ is the transmission coefficent TC $=A(t, y) / A_{\text {wavemaker }}\left(t_{\text {wavemaker }}, y\right)$ shown in Figure 5 (e). The value of the transmission coefficients calculated by using this somewhat heuristic method, which vary from 0.2 to 0.3 , are obtained for the range of crest locations from $y / \delta_{\omega}=2$ to 12 .

\section{Summary and Conclusion}

This paper used direct numerical simulation to examine the waves on the instability of the unbounded mixing layer in shallow waters without making an a priori assumption for the normal mode. The simulation for the subcritical instability has produced the anisotropic structure of the instability and its dependence on the convective Froude number. These results could also have been obtained from the solution of the eigenvalue problem. The simulation for the supercritical instability, on the other hand, has produced the modulating growth of the trapped waves between and beyond the returning surfaces. The amplitude modulation due to the group of waves is not an eigenvalue problem describable by the classical method.

\section{References}

Balmforth, N. J. 1999. Shear instability in shallow water. J. Fluid Mech. 387, 97-127.

Blumen, W., Drazin, P. G. \& Billings, D. F. 1975. Shear layer instability of an inviscid compressible fluid. part 2. J. Fluid Mech. 71 (2), 305-316.

Criminale, W. O., Jackson, T. L. \& Joslin, B. D. 2003. Theory and Computation of Hydrodynamic Stability. Cambridge University Press.

Karimpour, S. \& Chu, V. H. 2015a. High-order interpolation schemes for shear instability simulations. Intl. J. Numerical Methods for Heat \& Fluid Flow 25 (6), 1340-1360.

Karimpour, S. \& Chu, V. H. 2015b. Shear instability in high-speed shear flow. Proceedings of the 22nd Canadian Hydrotechnical Conference, Canadian Society of Civil Engineers pp. 1-10.

Liggett, J. A. 1994. Fluid mechanics. McGraw-Hill Higher Education.

Lindzen, R. S. \& Barker, J. W. 1985. Instability and wave over-reflection in stably stratified shear flow. J. Fluid Mech.151, 189-217. 
Lindzen, R. S., Farrell, B. \& Tung, K. K. 1980. The concept of wave overreflection and its application to baroclinic instability. J. Atmospheric Sciences 37 (1), 44-63.

Mack, L. M. 1990. On the inviscid acoustic-mode instability of supersonic shear flows. Theoretical and Computational Fluid Dynamics 2 (2), 97-123.

Maslowe, Sherwin A 1991. Barotropic instability of the bickley jet. J. Fluid Mech. 229, 417-426.

Sandham, N. D. \& Reynolds, W. C. 1990. Compressible mixing layer-linear theory and direct simulation. AIAA journal 28 (4), 618-624.

Schmid, P. J. \& Henningson, D. S. 2012. Stability and transition in shear flows. Vol. 142, Springer Science \& Business Media.

Takehiro, S. I. \& Hayashi, Y. Y. 1992. Over-reflection and shear instability in a shallow-water model. J. Fluid Mech. 236, 259-279.

Trefethen, L. N., Trefethen, A. E., Reddy, S., Driscoll, T. et al. 1993. Hydrodynamic stability without eigenvalues. Science 261 (5121), 578-584. 
Table 1. The disturbance's amplitude over the linear development from time $t=t_{b}$ to $t_{e}$.

\begin{tabular}{c|c|c|c|c|c|c|c|c}
$\operatorname{Fr}_{\mathrm{c}}$ & $k_{x}$ & $\sqrt{\overline{\bar{K}^{\prime}}}{ }_{b} /\left(U_{1}-U_{2}\right)$ & $t_{b} \hat{U}_{y}$ & $\sqrt{\overline{\overline{K^{\prime}}}} /\left(U_{1}-U_{2}\right)$ & $t_{e} \hat{U}_{y}$ & $\alpha / \hat{U}_{y}$ & $T_{M} \hat{U}_{y}$ & $T \hat{U}_{y}$ \\
\hline (a) $\operatorname{Fr}_{\mathrm{c}}=0.95$ & 0.40 & $2 \mathrm{E}-9$ & 20 & 0.36 & 500 & 0.0407 & - & - \\
(b) $\mathrm{Fr}_{\mathrm{c}}=1.20$ & 0.35 & $2 \mathrm{E}-9$ & 90 & 0.23 & 1450 & 0.0130 & 79 & 30 \\
(c) $\mathrm{Fr}_{\mathrm{c}}=1.40$ & 0.35 & $2 \mathrm{E}-9$ & 250 & 0.313 & 2250 & 0.0094 & 55 & 27
\end{tabular}


Fig. 1. (a) TANH base-flow velocity profile. The thick arrows are the velocity vectors at the returning surfaces where the current speed matches the gravity-wave speed. (b) The periodic boundary condition is defined at $x=0$ and $x=\lambda_{x}$, and the radiation boundary at $y=y^{-}$and $y=y^{+}$. The contours define the velocity fluctuations $u^{\prime}$ for $\operatorname{Fr}_{\mathrm{c}}=1.4$. The dotted dashed lines mark the location of the returning surfaces. The dot-dot line shows the wave crest of the oblique waves on one side of the free stream. (c) The same contours of $u^{\prime}$. The dot-dot line is the oblique wave crest on the other side of the free stream.

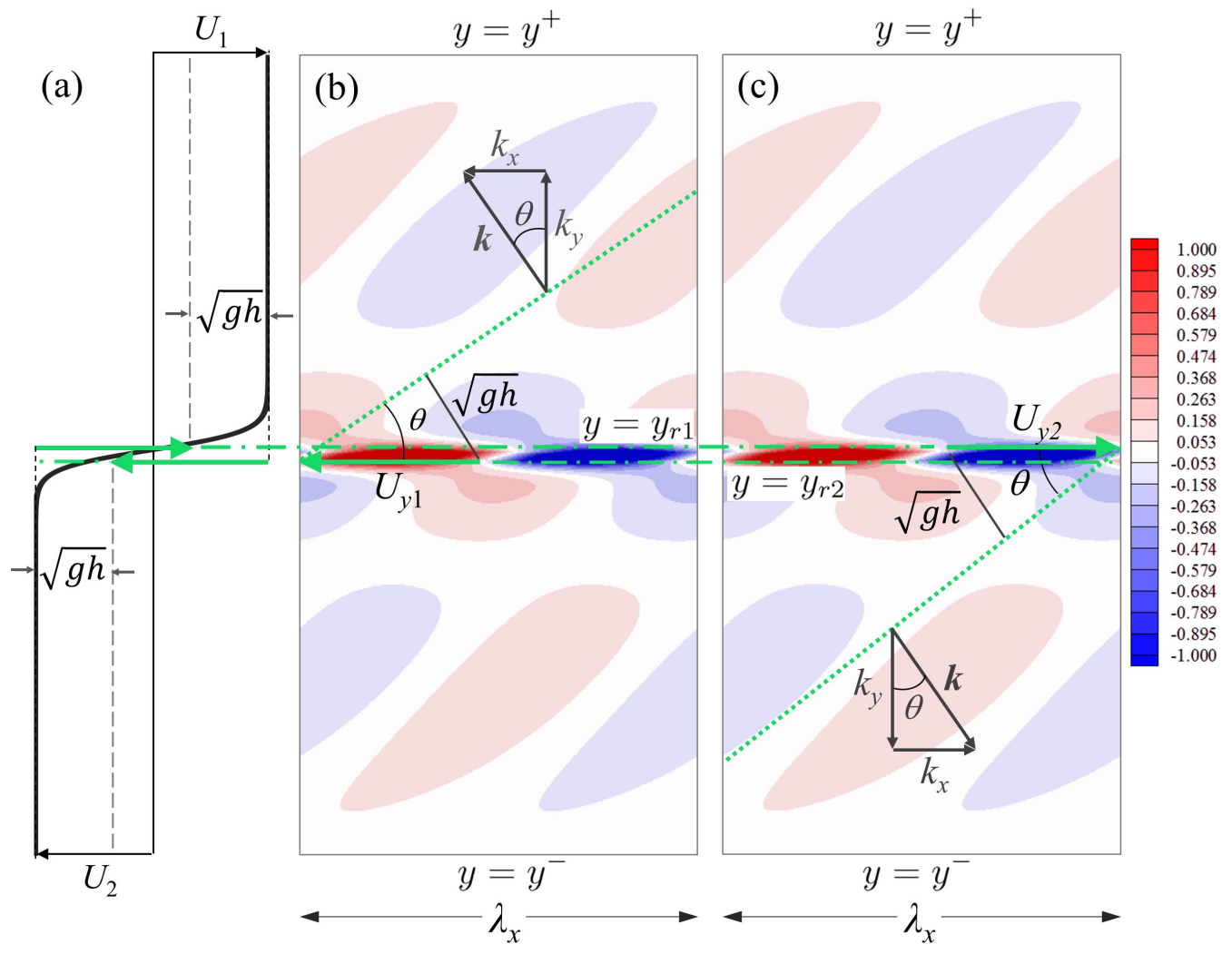


Fig. 2. The growth of the disturbance's kinetic energy, $\overline{\overline{K^{\prime}}} /\left(U_{1}-U_{2}\right)$, with time $t \hat{U}_{y}$ on the semilogarithmic scale for (a) $\mathrm{Fr}_{\mathrm{c}}=0.95, k_{x}=2 \pi / \lambda_{x}=0.40$ (b) $\mathrm{Fr}_{\mathrm{c}}=1.2, k_{x}=0.35$ and (c) $\mathrm{Fr}_{\mathrm{c}}=1.4, k_{x}$ $=0.35$.
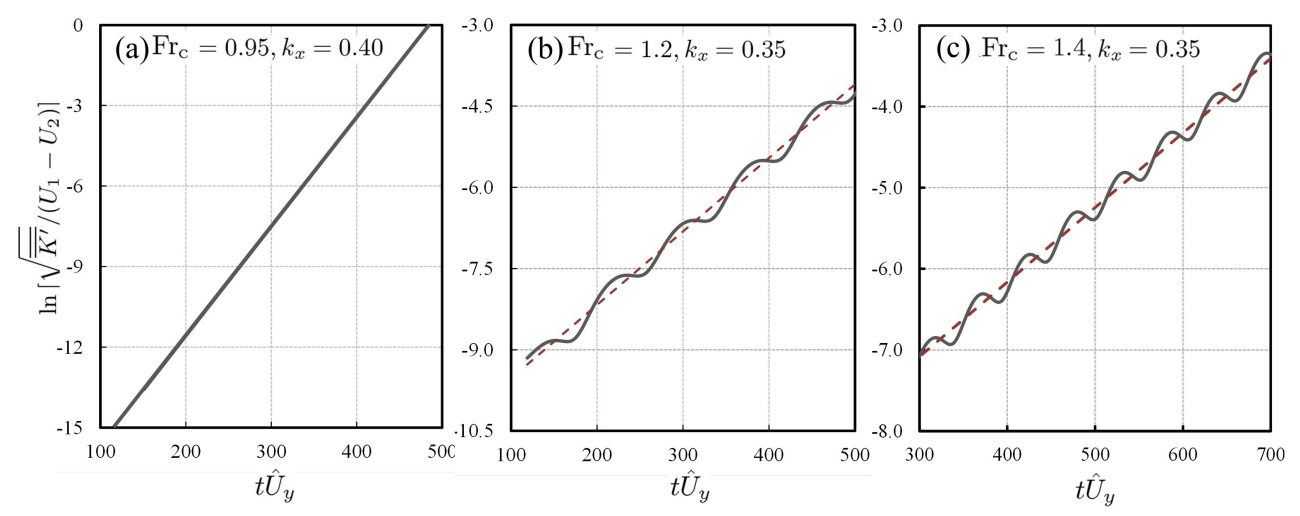
Fig. 3. (a) Fractional growth rate of the sub-critical instability obtained from the direct numerical simulation for convective Froude numbers $\mathrm{Fr}_{\mathrm{c}}=0.1,0.8$ and 0.95. The solid lines are the rates for the convective Mach number $\mathrm{Ma}_{\mathrm{c}}=0.01$ and 0.8 obtained by Sandham and Reynolds (1990) for ideal gas. (b) Averaged fractional growth rate of the supercritical instability for convective Froude numbers $\operatorname{Fr}_{\mathrm{c}}=1.1,1.2$ and 1.4. The dashed line is the rate for Mach number $\mathrm{Ma}_{\mathrm{c}}=1.2$ obtained by Sandham and Reynolds (1990).
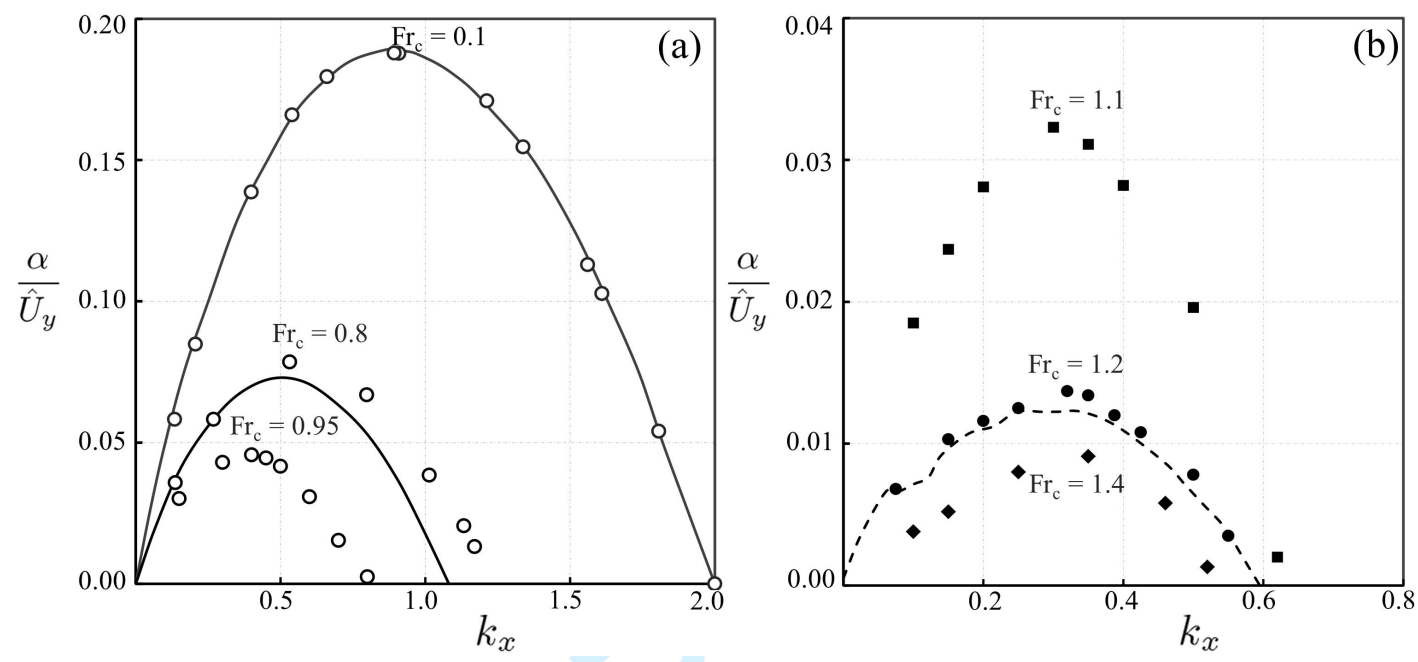
Fig. 4. (a) The constant structure for the subcritical instability with $\operatorname{Fr}_{\mathrm{c}}=0.1, k_{x}=0.90$. ( $\left.\mathrm{b}_{1}\right)$ and $\left(\mathrm{b}_{2}\right)$ The consistent structure for the supercritical instability with $\mathrm{Fr}_{\mathrm{c}}=1.40$ and $k_{x}=0.35$ over the modulation period at time $t=\frac{1}{4} T_{M}$ and at time $t=\frac{3}{4} T_{M}$. The vertical dotted dashed lines in $\left(\mathrm{b}_{1}\right)$ and $\left(\mathrm{b}_{2}\right)$ mark the returning surfaces at $y / \delta_{\omega}= \pm 0.147$.
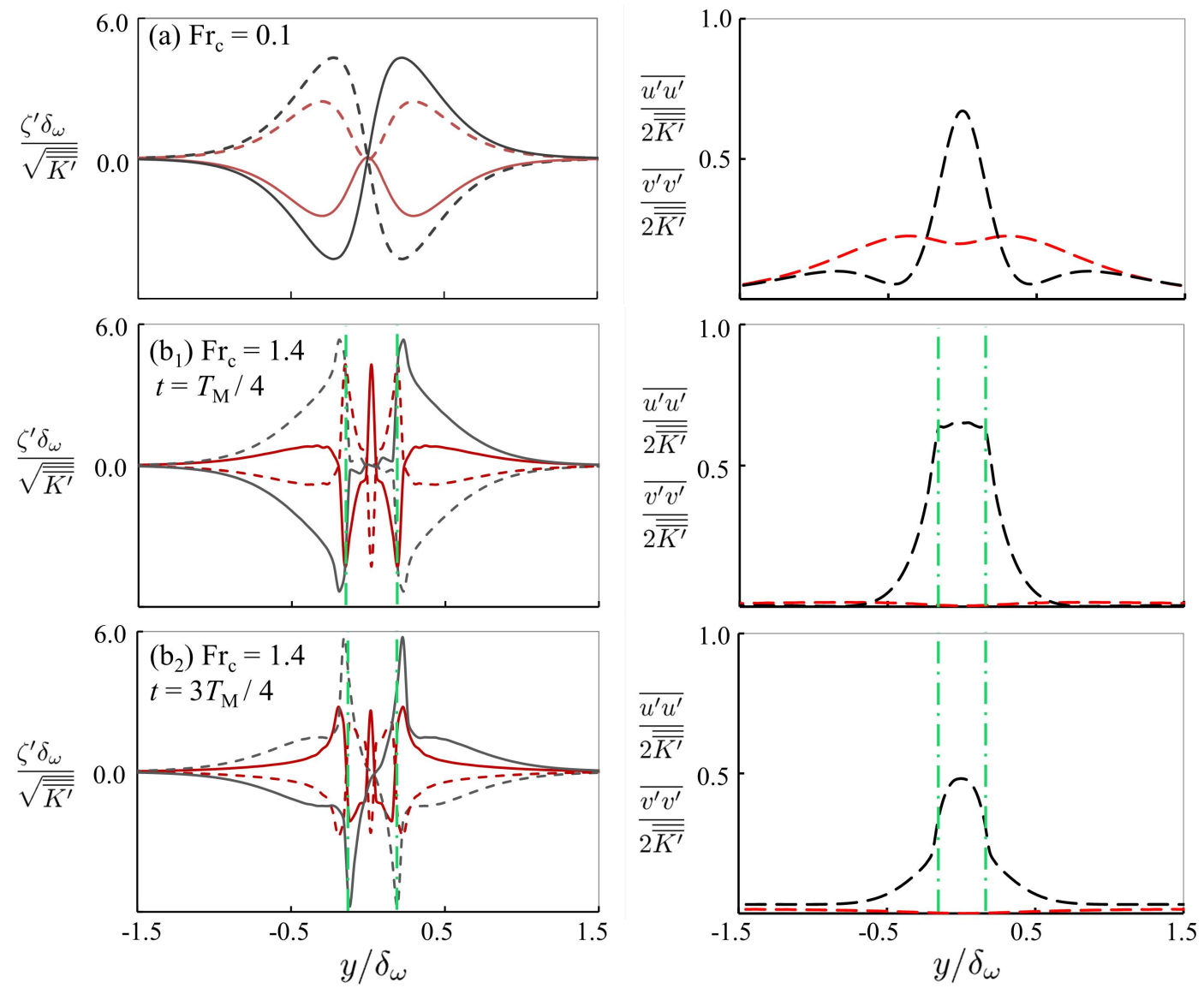
Fig. 5. The velocity fluctuation $u^{\prime} / \sqrt{\overline{\overline{K^{\prime}}}}$ profiles for $x=\lambda_{x} / 4, \lambda_{x} / 2,3 \lambda_{x} / 4$ and $\lambda_{x}$ over the modulation period at time (a) $t=T_{M} / 4$, (b) $t=T_{M} / 2$, (c) $t=3 T_{M} / 4$, (d) $t=T_{M} ; \mathrm{Fr}_{\mathrm{c}}=1.2$ and $k_{x}=0.35$. The dotdot lines define the envelope of the waves. The dotted dashed lines mark the location of returning surfaces at $y / \delta_{\omega}= \pm 0.084$. (e) Transmission coefficient.

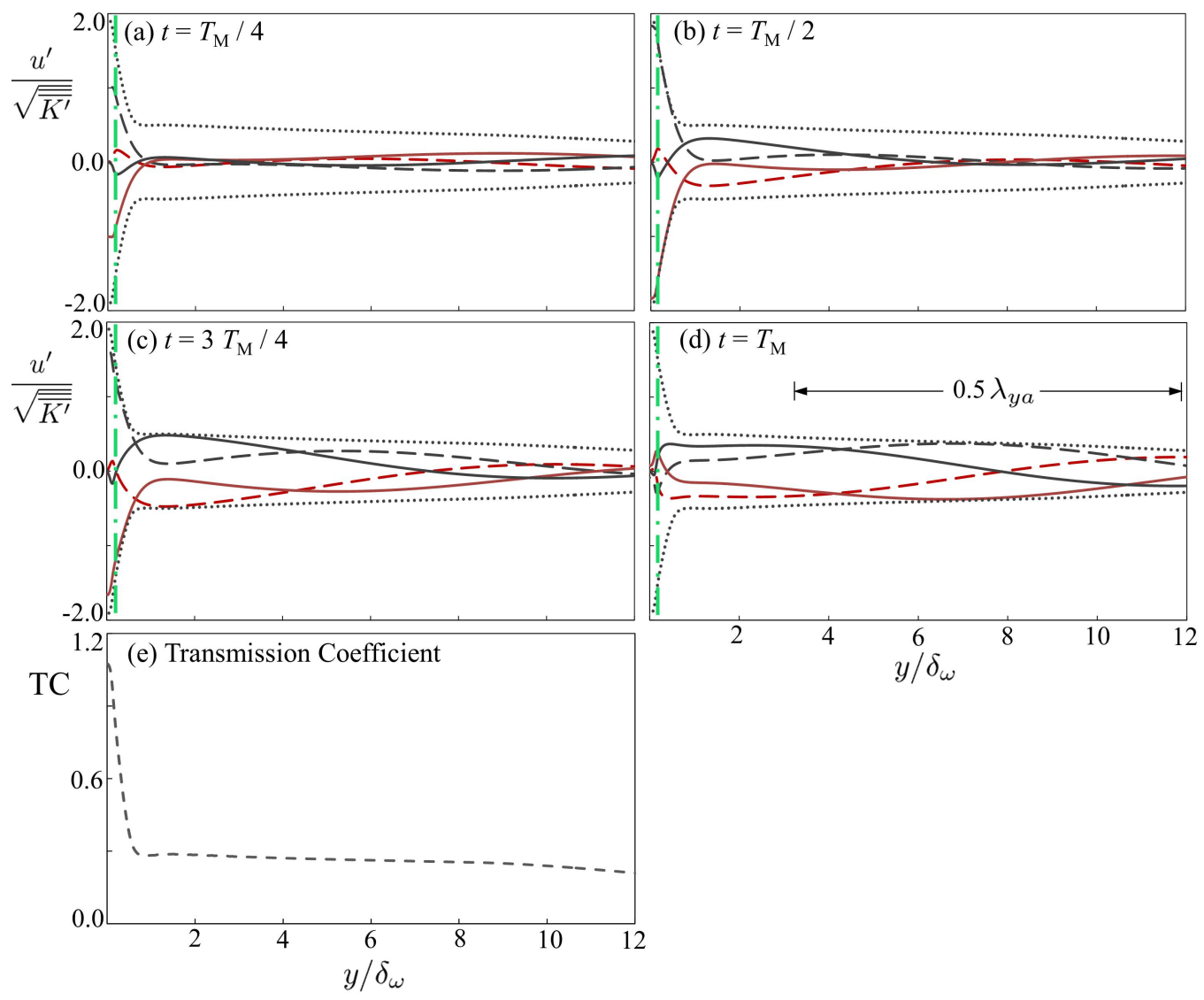


Fig. 6. The angle of the oblique waves in the free streams obtained from the simulations compared with (a) the correct formula derived from the correct hypothesis and (b) the incorrect formula.
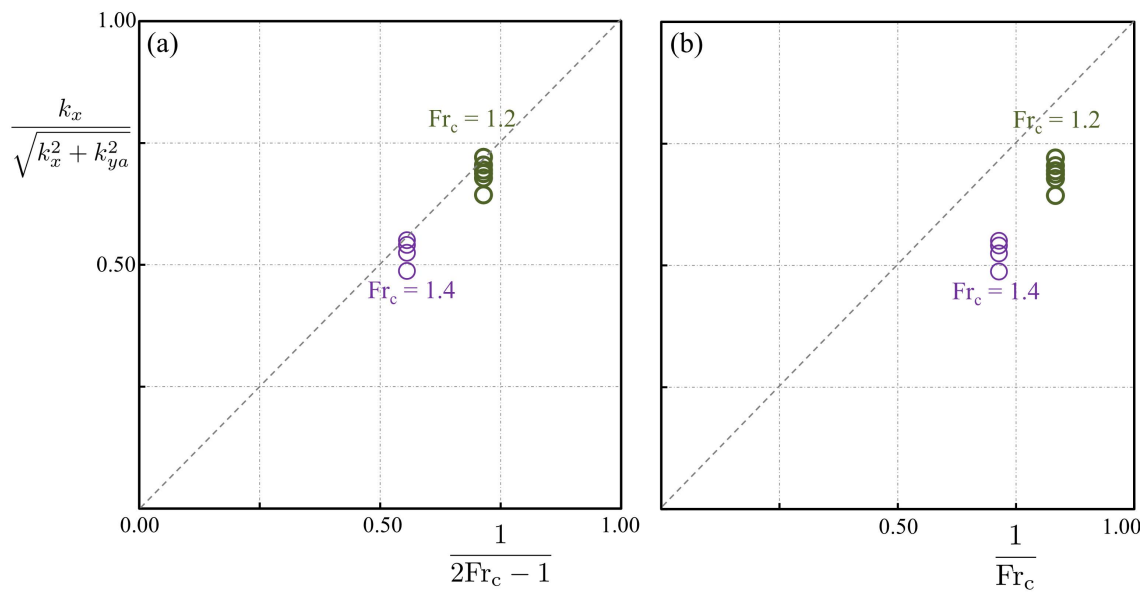
Fig. 7. Dependence of (a) $\overline{\overline{P^{\prime}}} / H \overline{\overline{K^{\prime}}}$, (b) $\overline{\overline{v^{\prime} v^{\prime}}} / \overline{\overline{u^{\prime} u^{\prime}}}$ and (c) $\overline{\overline{u^{\prime} v^{\prime}}} / \overline{\overline{K^{\prime}}}$ on the convective Froude number.
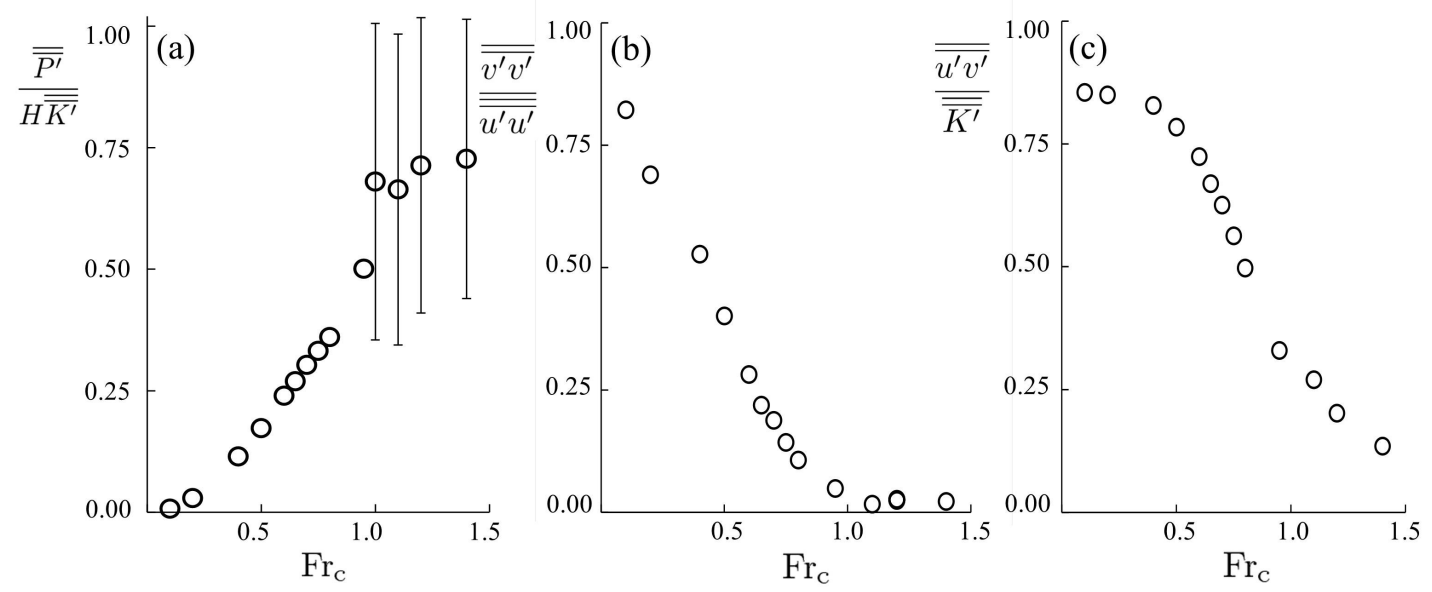

Published by NRC Research Press 